P147 (continued)

1 vs $42.2 \%+40 \%$ school 2 ; vegetable consumed: $67 \%+$ $44 \%$ school 1 vs $18 \%+32 \%$ school 2 ).

Conclusions and Implications: The protocol resulted in reliable estimation of FV intake using digital photography. Being able to accurately estimate FV intake will benefit intervention development and evaluation.

Funding: University of Illinois Extension

\section{P148 Nutrition Education in the Classroom Utilizing MyPlate Improves Children's Knowledge of Food Groups and Preferences in Kindergarten}

Tomoko Osera,PhD, tomokocup12@yahoo.co.jp, Hygiene and Preventive Medicine, Graduate School of Life Science, Kobe Women's University, 4 Chrome-7-2 Minatojima Nakamachi, Chuo Ward, Kobe, Hyogo Prefecture 650-0046, Japan; S. Tsutie, MS; Y. Segawa, MS; M. Kobayashi, MS, Takakuradai Kindergarten at Kobe Women's University; N. Kurihara, PhD, Kobe Women's University

Objective: To examine the effects of a nutrition education program using MyPlate on food group knowledge and food preference in kindergarten age children.

Target Audience: A total of 80 children (mean $5.1 \pm 0.2$ ages) attending kindergarten participated in the study.

Theory, Prior Research, Rationale: The provision of knowledge does not necessarily lead to a healthy lifestyle. Knowledge is the first step to change behavior. Teachers and nutritionists provided information about food groups to children using MyPlate.

Description: A quasi-experimental pre- post-test design was used to examine the effect of the nutrition education program. Both the intervention group $(n=55)$ and comparison group $(n=25)$ received basic nutrition education from their teachers that included gardening and cooking at least once a month. Children in the intervention group received an additional 10 minutes of nutrition education with MyPlate during lunchtime, which was held 4 times/week for 16 weeks.

Evaluation: The survey assessing children's food preferences was completed by their parents and the survey assessing children's knowledge about food groups was completed by the children. A measurement scale was used for dichotomous responses. Based on food preference, the number of disliked foods was significantly less in the intervention group compared with the comparison group $(\mathrm{p}<0.05)$. The improvement in knowledge about food groups in the intervention group was significantly higher than the comparison group $(\mathrm{p}<0.001)$.

Conclusions and Implications: Nutrition education involving the MyPlate program is suggested to increase knowledge about food groups and improve food preference in kindergarten age children. This program may be useful for changing children's food preferences in kindergarten age children.

Funding: None
P149 Knowledge, Attitudes and Practices of Nutrition and Physical Activity in Children of Primary Schools in Chiapas, Yucatan and Quintana Roo, Mexico

Mariana Perez, MA, mariana.perez@savethechildren.org, Save the Children, Francisco Sosa No. 30 Col. Villa, Coyoacan Del. Coyoacan, Mexico, DF C.P 04000; L. Saltijeral, $M A$

Objective: To identify the knowledge, attitudes and practices of nutrition and physical activity in children of primary schools in Chiapas, Quintana Roo and Yucatan, Mexico.

Design, Setting, and Participants: The diagnosis was conducted in October 2015 in 36 primary schools (19 in Chiapas, 4 in Quintana Roo and 13 in Yucatan). A Knowledge, Attitudes and Practices survey (KAP) was applied to a statically significant randomized sample of 1292 students from grades 4 th through 6 th. The data analysis was descriptive.

Results: Forty-nine percent of the respondents were boys and $51 \%$ girls. The analysis shows, $69 \%$ of the children reported plain water consumption at school the day before the survey, and that $15 \%$ had drank three or more sugarsweetened beverages; $70 \%$ had consumed vegetables and $66 \%$ had consumed at least one fruit. However, 43\% consumed fried foods and 51\% sweets or pastries, the day prior to the evaluation. Concerning the practice of physical activity (PA), 97\% of respondents considered it as favorable to keeping a healthy weight and 95\% said that being overweight affects health; however, only $28 \%$ performed at least 30 minutes of PA three times a week.

Conclusions and Implications: It is necessary to make greater efforts to promote the practice of PA in school. Educational strategies focused on the modification of eating habits and PA for health, should build on knowledge and attitudes prevalent in the school population, in order to ensure the success of educational interventions. Funding: FEMSA

\section{P150 National Competitive Foods Standards in Rural Virginia Middle Schools: Smarter Snacking or Compliance Lacking?}

Georgianna Mann, PhD, gmann89@vt.edu, Virginia Tech, 338 Wallace Hall, Blacksburg, VA 24061; V. Kraak, PhD, RD; E. Serrano, PhD

Objective: This study examined compliance of foods and beverages available for purchase by students in vending machines and à la carte to evaluate compliance in rural Appalachian Virginia middle schools before and after implementation of the Smart Snacks in School federal regulation.

Design, Setting, and Participants: Audits of food and beverage products sold in vending machines and à la carte were completed in spring of 2014 and 2015 in eight middle 